\title{
A large multisite cancer family is linked to BRCA2
}

\author{
P Tonin, P Ghadirian, C Phelan, G M Lenoir, H T Lynch, F Letendre, D Belanger, \\ $M$ Monté, S A Narod
}

\begin{abstract}
We identified a large French-Canadian family with 21 cases of breast cancer, including two affected brothers. Segregation of markers from chromosome $13 q$ in this family showed linkage to the BRCA2 gene locus (lod $=3 \cdot 67$ at D13S289). A number of cancers of other types occurred in this family, including three cases of prostate cancer and two cases of lymphoma. The penetrance of breast cancer among BRCA2 carriers is estimated to be $\mathbf{7 5 \%}$ to the age of 70 .
\end{abstract}

(f Med Genet 1995;32:982-984)

The majority of families with a dominant predisposition to breast cancer may be attributed to mutations in either the BRCAl gene on chromosome 17 or the BRCA2 gene on chromosome 13q. The BRCA1 gene was cloned in $1994^{1}$ and predictive testing by direct mutation analysis is now available. BRCA1 accounts for the majority of families with the breast-ovarian cancer syndrome ${ }^{2}$ but few families with cases of male breast cancer are linked to this gene. ${ }^{3}$ A second breast cancer locus, BRCA2, was mapped to chromosome $13 \mathrm{q}$ in $1994 .{ }^{4}$ The mapping of the BRCA2 gene was achieved by the application of a genomic search process to 15 extended families with multiple cases of breast cancer which had previously been shown not to be linked to the BRCA1 locus. Cases of male breast cancer appeared in six of the 15 families. BRCA2 mapped to the interval between D13S289 and D13S267, close to the marker D13S260. Overall, it was estimated that $74 \%$ of the 15 families were linked to BRCA2 and five of six families with male breast cancer gave positive lod scores to markers in the region. In order to confirm the association of hereditary male breast cancer and the BRCA2 locus, and to characterise further the range of cancers associated with this gene, we have typed a large French-Canadian cancer family with six polymorphic markers from chromosome 13q.

\section{Materials and methods}

This family was identified in the course of a case-control study of familial risk factors in cancer conducted at the Hotel Dieu Hospital in Montreal. ${ }^{5}$ Blood for DNA analysis was obtained from 58 people. Lymphocyte DNA was typed with six highly polymorphic CA repeat probes from chromosome 13q: AFM238zd9, D13S290, D13S289, D13S260, D13S171, and D13S267. ${ }^{4}$ BRCA2 is located between D17S289 and D17S267. The relative position of BRCA2 and D17S260 is not yet known. Lod scores were calculated using the LINKAGE program. ${ }^{6}$ Breast cancer susceptibility was modelled as an autosomal dominant trait with incomplete penetrance. Men with breast cancer were assumed to be carriers of the BRCA2 mutation. Cancers at sites other than the breast were nct used to define the individual phenotypes. The six CA repeat loci were analysed as systems of nine equally frequent alleles.

\section{Results}

The pedigree and genetic analysis of this family are shown in the figure. The average age at diagnosis of the 19 female breast cancers was 46.9 years. Two cases of male breast cancer were diagnosed in brothers at ages 64 and 43 .

The two point lod score at the D13S289 locus was $3 \cdot 67$. This lod score is equivalent of odds of 4000 to one in favour of linkage, and corresponds to a posterior probability of linkage of $>99 \%$. By inspection of the segregating haplotypes it is seen that both male cases of breast cancer and 14 female breast cancer cases carry all or part of the $(5,8,6,4,6,3)$ risk haplotype. Genotype information was not available for two affected women who had died. Subjects 9 and 10 had breast cancer diagnosed at ages 43 and 55 respectively, but do not carry the BRCA2 at risk haplotype. These two are likely to be sporadic cases.

Three confirmed cases of prostate cancer were observed in this family at ages 54,58 , and 71 years. Two of these (subjects 164 and 173) carry the BRCA2 risk haplotype and the genotype for the third case is not known. Other cases of cancer include two cases of lymphoma (subjects 239 and 114), carcinoma of the ampulla of Vater (subject 107), and cancers of the pancreas (subject 222), larynx (subject 275), and colon (subject 64). Two reported cancers 


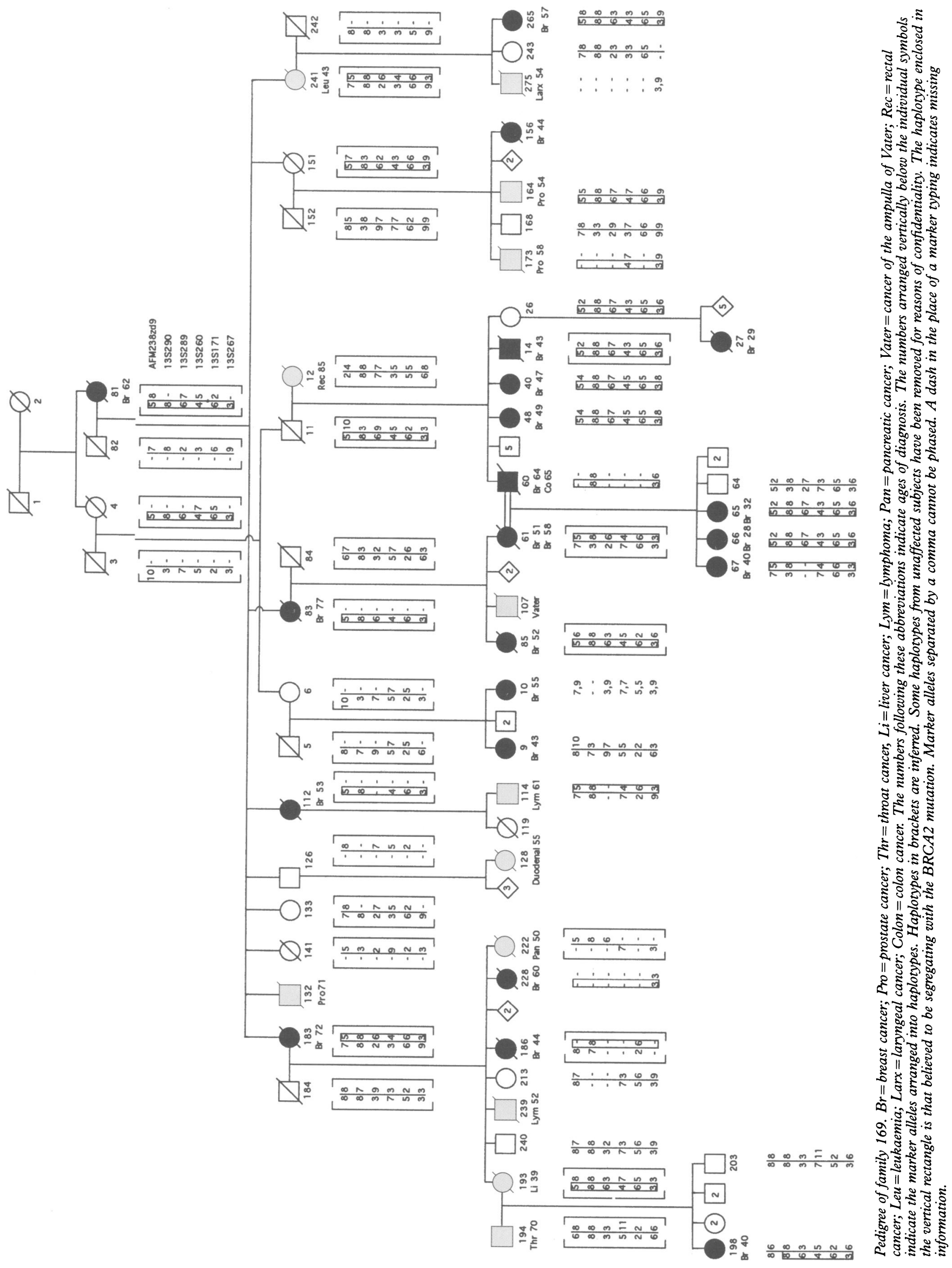


were not confirmed; liver cancer in subject 193 and leukaemia in subject 214 . One of the two patients with lymphoma, as well as the patients with leukaemia, liver cancer, colon cancer, and laryngeal cancer appear to be carriers of the BRCA2 gene mutation; the genetic status for the other three cancer cases is not known. The two cases of breast cancer appear to be the only cases identified among non-carriers (sporadic cancers) in this family.

\section{Discussion}

This large French-Canadian kindred confirms the assignment of a breast cancer susceptibility gene to chromosome $13 \mathrm{q}$, as reported by Wooster et $a l^{4}$ in 1994. Male breast cancer is a characteristic of about one-half of the BRCA2 linked families reported to date. Our family is unusual because of the marriage between subjects 60 and 61 , both of whom have had breast cancer, and who appear to have inherited the same BRCA2 mutation from a common great grandparent. All three of their daughters have had early onset breast cancer, although none is homozygous for the BRCA2 mutation.

We also follow a second, large, site specific breast cancer family (IARC family 2932) which was included in the original report of Wooster et $\mathrm{al}^{4}{ }^{4}$ The lod score for linkage of family 2932 to the D13S267 marker was $2 \cdot 05$. Family 2932 contains 13 cases of invasive breast cancer (mean age $45 \cdot 7$ years) as well as two cases of lung cancer, two of testicular cancer, and single cases each of stomach, prostate, brain, melanoma, and non-melanoma skin cancer. There are no male breast cancers in this family. A case of seminoma occurred in a 55 year old BRCA2 carrier. The other testicular cancer (embryonal cell carcinoma at the age of 22) was in a non-carrier. In the two families com- bined, the penetrance of breast cancer was $42 \%$ by age 50 and $75 \%$ by age 70 . Although a moderately increased risk of ovarian cancer has been reported in BRCA2 carriers, ${ }^{4}$ none of the 48 BRCA2 carriers in either of the two pedigrees is affected with ovarian cancer.

It appears that many of the BRCA1 mutations in Canada are recurrent and are the result of common ancestors. ${ }^{7}$ It is likely that the frequencies of mutant alleles of BRCA1 and BRCA2 (and the ratios of BRCA1 to BRCA2 linked families) will differ between ethnic groups. This is the first reported example of a BRCA2 linked French-Canadian family; it is not yet clear if this is the predominant form of hereditary breast cancer in this population. It will be important to establish the relative contributions of BRCA1 and BRCA2 to hereditary cancer in different ethnic populations if predictive testing programmes are to operate efficiently.

This work was supported by the Department of the Army grants, DAMD17-94-J 4299, the Fonds de la Recherche en grants, DAMD17-94-J 4299, the Fonds de la Recherche en Santé du Quebec (FRSQ), and the Canadian Genetics Disease Network. We thank France Dion, Kenneth Morgan, Chantal
Perret, Deborah Lambert, and Jean Latreille for technical exPerret, Deborah Lambert, and
pertise and helpful discussions.

1 Miki Y, Swensen J, Shattuck-Eidens D, et al. A strong candidate gene for the breast and ovarian cancer susceptibility didate gene for the breast and ovarian canc

2 Narod SA, Ford D, Devilee P, et al. An evaluation of genetic heterogeneity in 145 breast-ovarian cancer families. Am $\mathcal{f}$ heterogeneity in 145 breast-

3 Stratton MR, Ford D, Neuhausen S, et al. Familial male Stratton MR, Ford D, Neuhausen S, et al. Familial male
breast cancer is not linked to the BRCA1 locus on chrobreast cancer is not linked to the BRCA1
mosome 17q. Nature Genet 1994;7:103-7.

4 Wooster R, Neuhausen S, Mangion J, et al. Localisation of a breast cancer susceptibility gene (BRCA2) to chromosome $13 q$ by genetic linkage analysis. Science 1994;265:2088-90. 5 Ghadirian P, Narod S, Perret C, et al. Hereditary breast cancer in 19 females and 2 males: kindred PG1940. Eur $\mathcal{F}$ Cancer 1995;31A:284-7.

6 Lathrop GM, Lalouel JM, Julier C, Ott J. Strategies for multilocus linkage analysis in humans. Proc Natl Acad Sci USA 1994;81:3343-6.

7 Simard J, Tonin P, Durocher F, et al. Common origins of BRCA1 mutations in Canadian breast and ovarian cancer families. Nature Genet 1994;8:392-8. 